\title{
Biomarker-enhanced triage in respiratory infections: a proof-of-concept feasibility trial
}

\author{
Werner C. Albrich 1,2,9, Kristina Rüegger ${ }^{1,9}$, Frank Dusemund ${ }^{1,3}$, Philipp Schuetz', \\ Birsen Arici', Alexander Litke ${ }^{1}$, Claudine A. Blum¹, Rita Bossart ${ }^{4}$, \\ Katharina Regez ${ }^{4}$, Ursula Schild ${ }^{4}$, Merih Guglielmetti ${ }^{4}$, Antoinette Conca ${ }^{4}$, \\ Petra Schäfer ${ }^{4}$, Maria Schubert ${ }^{5}$, Sabina de Geest ${ }^{5}$, Barbara Reutlinger ${ }^{4}$, \\ Sarosh Irani ${ }^{6}$, Ulrich Bürgi ${ }^{7}$, Andreas Huber ${ }^{8}$ and Beat Müller ${ }^{1}$
}

Affiliations: 'Medical University Dept, University of Basel, Kantonsspital Aarau, Aarau, ${ }^{2}$ Division of Infectious Diseases and Hospital Epidemiology, Kantonsspital St. Gallen, St. Gallen, ${ }^{3}$ Division of Pneumology, Kantonsspital St. Gallen, St. Gallen, ${ }^{4}$ Dept of Clinical Nursing Science, Kantonsspital Aarau, Aarau, Institute of Nursing Science, University of Basel, Basel, ${ }^{6}$ Dept of Pneumology, Kantonsspital Aarau, Aarau, ${ }^{7}$ Emergency Dept, Kantonsspital Aarau, Aarau, and ${ }^{8}$ Dept of Laboratory Medicine, Kantonsspital Aarau, Aarau, Switzerland. ${ }^{9}$ Both authors contributed equally to this work.

Correspondence: B. Müller, Medical University Dept, Kantonsspital Aarau, Tellstrasse, CH-5001 Aarau, Switzerland. E-mail: happy.muellerवunibas.ch

ABSTRACT Concerns about inadequate performance and complexity limit routine use of clinical risk scores in lower respiratory tract infections. Our aim was to study feasibility and effects of adding the biomarker proadrenomedullin (proADM) to the confusion, urea $>7 \mathrm{mmol} \cdot \mathrm{L}^{-1}$, respiratory rate $\geqslant 30$ breaths $\cdot \mathrm{min}^{-1}$, blood pressure $<90 \mathrm{mmHg}$ (systolic) or $\leqslant 60 \mathrm{mmHg}$ (diastolic), age $\geqslant 65$ years (CURB-65) score on triage decisions and length of stay.

In a randomised controlled proof-of-concept intervention trial, triage and discharge decisions were made for adults with lower respiratory tract infection according to interprofessional assessment using medical and nursing risk scores either without (control group) or with (proADM group) knowledge of proADM values, measured on admission, and on days 3 and 6. An adjusted generalised linear model was calculated to investigate the effect of our intervention.

On initial presentation the algorithms were overruled in 123 (39.3\%) of the cases. Mean length of stay tended to be shorter in the proADM ( $\mathrm{n}=154,6.3$ days) compared with the control group $(\mathrm{n}=159,6.8$ days; adjusted regression coefficient $-0.19,95 \%$ CI $-0.41-0.04 ; \mathrm{p}=0.1)$. This trend was robust in subgroup analyses and for overall length of stay within 90 days (7.2 versus 7.9 days; adjusted regression coefficient $-0.18,95 \%$ CI $-0.40-0.05 ; \mathrm{p}=0.13)$. There were no differences in adverse outcomes or readmission.

Logistic obstacles and overruling are major challenges to implement biomarker-enhanced algorithms in clinical settings and need to be addressed to shorten length of stay.

@ERSpublications

Proof-of-concept trial: how to shorten LOS in patients with LRTIs by reducing medically unnecessary days in the hospital http://ow.ly/nI7z4

For editorial comments see page 895.

Received: July 212012 | Accepted after revision: Dec 212012 | First published online: Jan 242013

Clinical trial: This study is registered at www.controlled-trials.com with identifier number ISRCTN62022490.

Support statement: Funding was provided by the Swiss National Science Foundation (grant number 32003B_135222/1; Dept of Health, Kanton Aargau; Forschungsrat and Medical University Department Kantonsspital Aarau, Aarau, Switzerland).

Conflict of interest: Disclosures can be found alongside the online version of this article at www.erj.ersjournals.com

Copyright OERS 2013. ERJ Open articles are open access and distributed under the terms of the Creative Commons Attribution Non-Commercial Licence 3.0.

This article was modified in April 2016 to correct errors in the licence information. 


\section{Introduction}

Community-acquired lower respiratory tract infections (LRTIs), including acute bronchitis, acute exacerbations of chronic obstructive pulmonary disease (AECOPD) and community-acquired pneumonia (CAP), are among the most frequent causes of hospitalisation [1]. Inpatient care of CAP is 8-20 times more costly than outpatient treatment [2,3], with higher risks of nosocomial complications [4]. Admission rates and length of stay (LOS) are variable in clinical routine and arbitrarily affected by medical, functional, psychosocial factors and patients' and relatives' preferences [2, 5-10]. In this context, scoring systems to quantify medical, nursing and social factors in a standardised way were developed. The self-care index (SPI; "Selbstpflegeindex") was developed to assess functional dependence in activities of daily living and predicts the need for social services [11]. The post-acute care discharge score (PACD) predicts the biopsychosocial risk, requirement for post-acute care and facilitates discharge planning [12]. In the UK and Scandinavia, nurse-led units (NLUs) have become popular institutional settings for patients with low medical and predominantly nursing-care needs $[13,14]$.

To standardise medical risk assessment in CAP, the confusion, urea $>7 \mathrm{mmol} \cdot \mathrm{L}^{-1}$, respiratory rate $\geqslant 30$ breaths $\cdot \mathrm{min}^{-1}$, blood pressure $<90 \mathrm{mmHg}$ (systolic) or $\leqslant 60 \mathrm{mmHg}$ (diastolic), age $\geqslant 65$ years (CURB-65) score is being propagated [15]. In previous research, we and others validated the feasibility of the CURB-65 score in CAP and, importantly, extended it to non-CAP LRTIs, i.e. acute bronchitis and AECOPD. Thereby, the CURB-65 score predicts mortality in patients with non-CAP LRTIs with similar prognostic accuracy as in CAP [16-18]. Limitations of clinical risk scores for triage decisions include their static behaviour over time, validation for predefined outcomes, considerable variability of outcomes within given risk categories and poor memorability [19].

Biomarkers are objective, dynamic and easily measurable. Proadrenomedullin (proADM) belongs to the calcitonin peptide superfamily and is ubiquitously hyperexpressed during bacterial infections. Of the biomarkers presently available, it currently has the best evidence for prognostic assessment in LRTIs [20-23]. We recently showed that proADM predicted mortality and complications in LRTIs similarly to the CURB-65 score and provided independent prognostic information within each CURB-65 risk class [16, 24]. As biomarkers and clinical scoring systems reflect different aspects of the host response, their combinations demonstrate superior prognostic accuracy [20, 25-27]. Combining proADM cut-off points with CURB-65 classes, we proposed a novel CURB-65-A score with improved prognostic accuracy for LRTI [16, 18]. Subsequently, we demonstrated the potential to reduce hospitalisations in patients with low medical risk (Optimised Patient Transfer using an Innovative Multidisciplinary Assessment (OPTIMA) I study) [28].

While low CURB-65 scores generally indicate the safety of outpatient therapy [29], additional clinical criteria improve its prognostic accuracy [24]. We hypothesised that triage decisions based on CURB-65 and medical stability criteria [30] are less effective than if these were enhanced by proADM. In this proof-ofconcept study, we tested the feasibility, possible effects and limitations of adding proADM to CURB-65, and clinical stability criteria and biopsychosocial scores (SPI and PACD) for initial risk stratification and subsequent clinical management for LOS. We compared results with the former observational OPTIMA I study (historical control group) [28].

\section{Materials and Methods \\ Study subjects and study design}

This was an interprofessional and pragmatic randomised controlled intervention trial that took place at an acute-care hospital and two post-acute centres in Switzerland between September 2010 and July 2011. The study was largely based on routine medical and nursing staff to closely reflect the clinical "real-life" setting in all patients in both groups. We consecutively enrolled patients (aged $\geqslant 18$ years) with community- or nursing home-acquired LRTI, including CAP, AECOPD, acute bronchitis and influenza. Exclusion criteria were inability to communicate in any of six commonly spoken languages, intravenous drug use, and either immediately life-threatening or terminal illness.

\section{Methods}

Patients were randomised 1:1 following a computer-generated randomisation scheme, stratified for type of LRTI, into a proADM or a control group. Patients were triaged by the treating physician according to an interprofessional medical and nursing risk assessment consisting of medical (CURB-65 on admission and medical stability criteria during hospitalisation), functional (SPI) and biopsychosocial criteria (PACD), either with (proADM group) or without knowledge (control group) of proADM values (fig. 1).

The triage algorithm is based on medical and biopsychosocial risk assessment with and without addition of proADM for patients with lower respiratory tract infection on admission. The medical overruling criteria were as follows. Admission to intensive care unit, based on respiratory (respiratory rate $\geqslant 30 \mathrm{~min}^{-1}$ and/or 


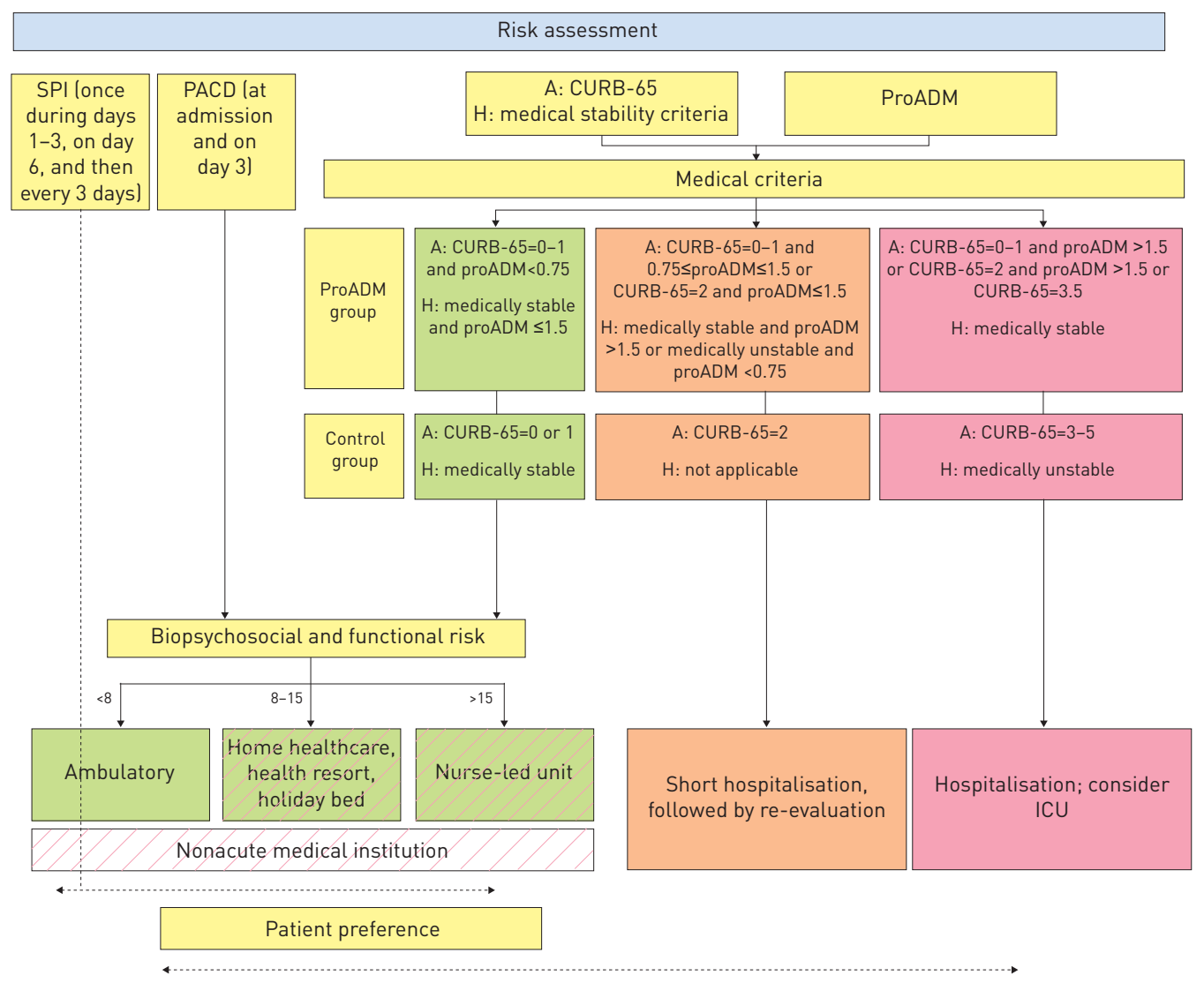

FIGURE 1 Algorithm of risk assessment for triage decisions on admission (A) and during hospitalisation (H). SPI: selbstpflegeindex (self-care index); PACD: post-acute care discharge; proADM: proadrenomedullin; CURB-65: confusion, urea $>7 \mathrm{mmol} \cdot \mathrm{L}^{-1}$, respiratory rate $\geqslant 30$ breaths $\cdot \mathrm{min}^{-1}$, blood pressure $<90 \mathrm{mmHg}$ (systolic) or $\leqslant 60 \mathrm{mmHg}$ (diastolic), age $\geqslant 65$ years; ICU: intensive care unit.

oxygen saturation $<90 \%$ with $6 \mathrm{~L} \cdot \mathrm{min}^{-1}$ of oxygen) or haemodynamic instability (systolic blood pressure for $\geqslant 1 \mathrm{~h}<90 \mathrm{mmHg}$ despite adequate volume resuscitation or vasopressor requirement); life-threatening comorbidity, i.e. imminent death; complications (abscess or empyema); for COPD patients, a Global Initiative for Chronic Obstructive Lung Disease score of III and IV; oxygen saturation $<90 \%$ despite 30 min of intensive treatment; acute illness requiring hospitalisation independent from LRTI; comorbidity, i.e. immunodeficiency (neutrophils $<500$ per $\mu \mathrm{L}$; if HIV+: CD4 $<350$ per $\mu \mathrm{L}$; leukaemia, lymphoma, myeloma, cytotoxic medications or haemodialysis), pneumonia within last 6 weeks; hospitalisation independent of indication within the last week; other significant lung disease (cancer, fibrosis, bronchiectasis, tuberculosis, pulmonary embolism or cavitary lung disease); and confusion, delirium or i.v. drug use. Biopsychosocial and functional overruling criteria were as follows. Criteria requiring intensive nursing care, i.e. dementia, recurrent falls, pressure ulcer and inability to reliably take medications; SPI score $<32$ points in patients with a low PACD score $(<8)$ [11]; and deficit of mobility or self-care requiring treatment. Organisational overruling criteria were as follows. Waiting for placement in a nonacute medical care facility (holiday bed, rehabilitation, nursing home or home healthcare); and waiting for laboratory results, imaging studies or consultant examinations. Patient's preference was defined as a patient's or relative's concerns about safety at home; a lack of supporting social network; or financial reasons. Medical stability during hospitalisation [31] included all of the following. Feasibility of oral intake without need for i.v. therapy; stable vital signs for $\geqslant 24 \mathrm{~h}$ (i.e. temperature $<37.8^{\circ} \mathrm{C}$; heart rate $\leqslant 100$ beats $\cdot \mathrm{min}^{-1}$; respiratory rate $\leqslant 24$ breaths $\cdot \mathrm{min}^{-1}$; oxygen saturation $\geqslant 90 \%$ or oxygen tension $\geqslant 60 \mathrm{mmHg}$ on breathing room air or, in patients with home oxygen therapy, no higher oxygen flow; and systolic blood pressure $\geqslant 90 \mathrm{mmHg}$ ); and mental status at level before LRTI.

Site of care was recommended in both groups as follows. Regular hospitalisation or intensive care unit (ICU) admission in patients at high medical risk; short hospitalisation for $48 \mathrm{~h}$ followed by re-evaluation in 
patients at intermediate medical risk; and ambulatory care, home healthcare, health resort, rehabilitation or NLU according to biopsychosocial risk for patients at low medical risk.

Predefined medical, biopsychosocial and organisational criteria and patient's preference could be used to optionally overrule triage decisions and transfer patients to higher risk classes. Biopsychosocial, functional and organisational criteria could increase the level of care up to the NLU. The patient's preference had priority for the final triage decision and patients remained blinded to study group assignment.

Hospitalised patients were assessed by registered nurses for medical stability criteria [29] three times daily until medical stabilisation. For the functional and biopsychosocial risk, the SPI [11] was assessed once within the first 3 days, and then on day 6 and every 3 days therafter; the PACD [12] score was documented on admission and day 3. Patients were considered appropriate for hospital discharge if they were medically stable without predefined medical, biopsychosocial, functional or organisational overruling criteria. In the proADM group, medical stability was additionally influenced by proADM levels on days 3 and 6 (fig. 1). Site of care was determined by biopsychosocial and organisational factors in patients who were otherwise appropriate for discharge.

Treating physicians were given formal introduction into correct application of proADM values, triage algorithms, including CURB-65, stability criteria and overruling criteria, and received regular reminders throughout the study. Nursing staff received on-going training on the correct use of biopsychosocial and functional criteria. The study team was permanently available for questions regarding the algorithm and oversaw the compliance with the triage pathways. Antibiotic therapy was provided according to previously validated and published procalcitonin (PCT) cut-off ranges [32].

All patients discharged to home received a telephone call within $24 \mathrm{~h}$ by a study nurse to confirm stability and address urgent problems. All patients underwent a standardised phone interview on days 30 and 90 by blinded members of the study team. The trial was approved by the local ethics committee (Kantonale Ethikkommission Aargau, Aargau, Switzerland; EKAG 2010/045) and was supervised by an independent data safety monitoring board.

\section{Methods of proADM measurement}

ProADM was measured in the central hospital laboratory from EDTA plasma with a commercially available immunoassay (MR-proADM, Thermofisher Scientific-BRAHMS AG, Hennigsdorf, Germany) with a functional assay sensitivity of $0.12 \mu \mathrm{g} \cdot \mathrm{L}^{-1}$ [20]. Results were routinely available within $1.5 \mathrm{~h}$ upon ordering around the clock and reported only for patients randomised to the proADM group.

\section{Definitions}

CAP, AECOPD, bronchitis and severity assessment of COPD were defined as described previously [16, 18]. Medical stability criteria for CAP were applied in all LRTIs [30]. Patients were considered medically stable if all of the following criteria were fulfilled. Feasibility of oral intake; stable vital signs for $\geqslant 24 \mathrm{~h}$ (i.e. temperature $<37.8^{\circ} \mathrm{C}$, heart rate $\leqslant 100$ beats $\cdot \mathrm{min}^{-1}$, respiratory rate $\leqslant 24$ breaths $\cdot \mathrm{min}^{-1}$, oxygen saturation $\geqslant 90 \%$ or oxygen tension $\geqslant 60 \mathrm{mmHg}$ on breathing room air; and systolic blood pressure $\geqslant 90 \mathrm{mmHg}$ ); mental status at level before LRTI; and no evidence of acute comorbidity necessitating hospitalisation.

Outpatient care was defined as discharge to home (either with or without home healthcare) from the emergency department. Adverse events were defined as any of the following. Mortality, ICU admission, recurrent infection and re-hospitalisation. LOS was defined as number of physician-led nights spent in hospital, i.e. excluding time spent in the NLU.

\section{End-points}

The primary end-point was overall LOS comparing the proADM with the control group. Secondary endpoints were comparisons with regard to measure of algorithm adherence, functional status, adverse events and readmission rates.

\section{Statistical analysis}

As a proof-of-concept study to primarily assess feasibility, the primary analysis population was the full analysis set, which included all randomised patients following an intention-to-treat principle. The primary analysis was repeated on the per-protocol-population, which excluded nonevaluable cases, violators of exclusion criteria and patients with final diagnoses other than LRTI (fig. 2).

We furthermore compared the results of the overall cohort to a previously published cohort from a prospective observational quality control study (OPTIMA I, historical control group), where triage decisions were recommended based on CURB-65 and medical stability criteria but were less strictly 


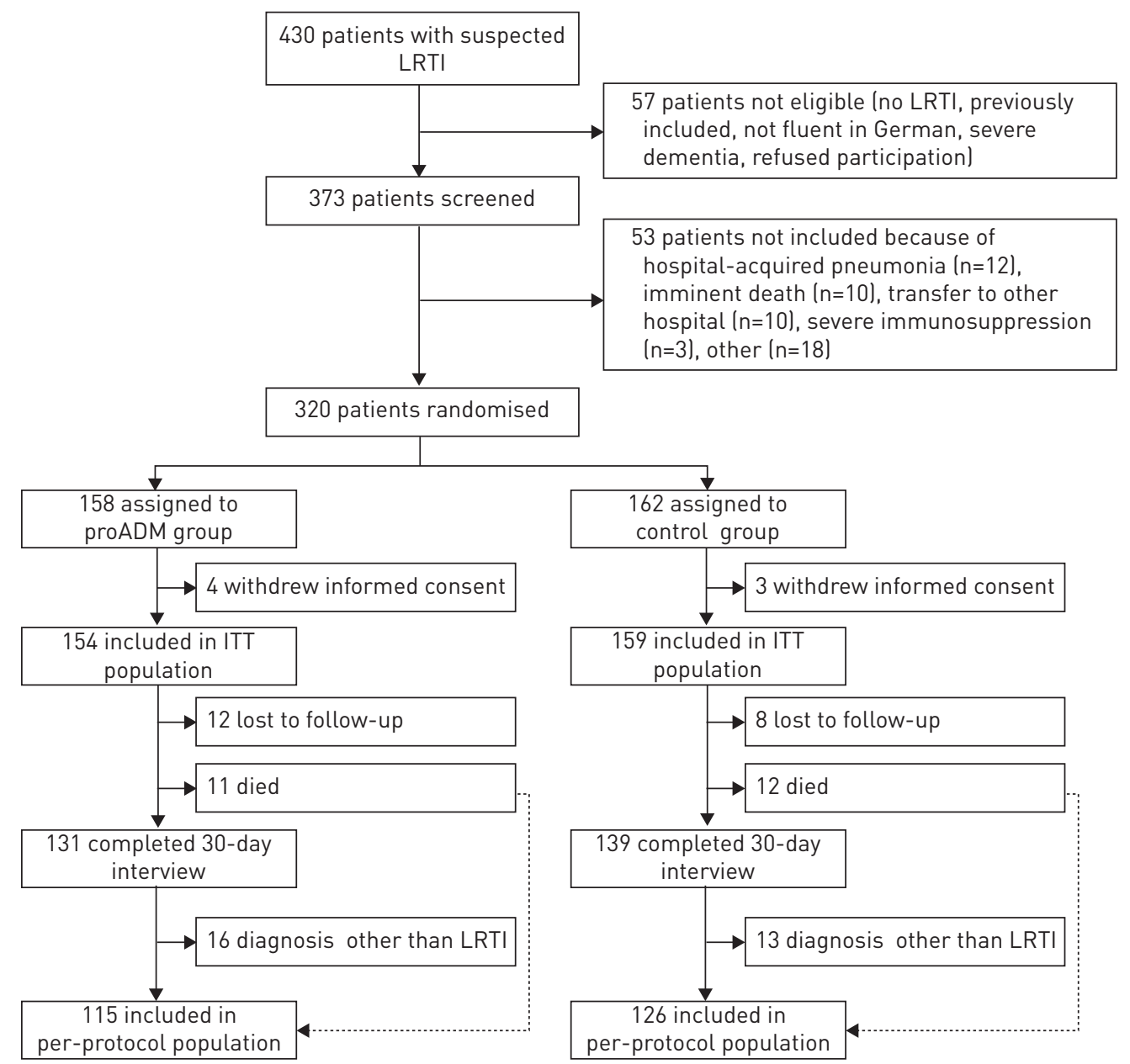

FIGURE 2 Flow diagram of patients in the trial. LRTI: lower respiratory tract infection; proADM: proadrenomedullin; ITT: intention-to-treat.

enforced by the study team [28]. This allowed us to assess the effect of more strictly enforcing triage pathways in the current intervention study compared with an historical control group, as surrogate for the presence and extent of a Hawthorne effect, i.e. improved triage performance by the treating physicians simply due to the knowledge of being monitored under study conditions.

The primary hypothesis of the randomised study was that knowledge of proADM values improves interdisciplinary risk assessment and safely reduces LOS without excess adverse events and patient dissatisfaction. Based on intention-to-treat analysis on the primary outcome, a power of $80 \%$, a two-sided $\alpha$ coefficient of $5 \%$, an expected mean $\pm S D$ LOS in the acute-hospital setting of $8 \pm 4$ days and expected difference of 1.5 days compared with an historical control [28], we expected to need a sample size of at least 113 patients per group. We planned to screen 350-400 and enrol 250-300 patients.

We used mean $\pm S D$, or median (interquartile range) to describe the population as appropriate. To investigate for difference between randomisation arms in regard to LOS, we calculated a generalised linear model with a gamma distribution as previously suggested for this type of outcome data [32]. We adjusted the model for the main predictors of LOS, namely age, sex, type of LRTI and the CURB-65 score. For secondary binary end-points, we calculated logistic regression models adjusted for the same set of covariates as described above. p-values were reported at the two-sided 5\% significance level.

\section{Results}

From a total of 430 screened patients with acute LRTI, we enrolled 313 (72.8\%) patients (fig 2). Baseline characteristics were balanced in both groups (table 1).

Overall, the algorithms were overruled in 123 (39.3\%) of the cases on initial presentation and in 108 (34.5\%) after hospital admission. In the control group, overruling occurred in 81 (50.9\%) patients on initial 


\section{TABLE 1 Baseline characteristics}

Control group

\section{Subjects}

Mean age years

Male $n$ (\%)

Initial treatment site $\mathbf{n}(\%)$

Inpatient treatment

Outpatient treatment

\section{Risk assessment}

CURB-65 mean, median

CURB-65 I

CURB-65 II

CURB-65 III

CURB-65-A class mean, median

CURB-65-A I

CURB-65-A II

CURB-65-A III

Confusion

Urea $>7 \mathrm{mmol} \cdot \mathrm{L}^{-1}$

Respiratory rate $\geqslant 30$ breaths $\cdot \mathrm{min}^{-1}$

Systolic blood pressure $<90 \mathrm{mmHg}$

Age $\geqslant 65$ years

Final diagnosis $\mathbf{n}(\%)$

Bronchitis

AECOPD

CAP

Influenza

Other

Coexisting illnesses \%

Lung cancer

Other cancer $<1$ year

Coronary heart disease

Mean Charlson comorbidity index

Anamnestic findings \%

Cough

Sputum

Dyspnoea

Tachypnoea

Chest pain

\section{Clinical findings}

Auscultatory findings $\%$

Fever \%

Shivering \%

Leukocytosis/leukopenia \%

Heart rate beats $\cdot \mathrm{min}^{-1}$

Temperature ${ }^{\circ} \mathrm{C}$

Laboratory findings

Mean proADM nM

On admission

Day 3

Day 6

Procalcitonin $\mu \mathrm{g} \cdot \mathrm{L}^{-1}$

Median on admission

$<0.25 \%$

$0.25-0.5 \%$

$>0.5 \%$

C-reactive protein $\mathrm{mg} \cdot \mathrm{L}^{-1}$

Leukocyte count cells $\cdot \mu \mathrm{L}^{-1}$

159
61.3
$94(59.1)$
$126(79.3)$
$33(20.7)$
$1.2,1$
102
33
24
$2,2^{\#}$
$48^{\#}$
$63^{\#}$
$48^{\#}$
11
62
22
4
74

$31(19.5)$

$22(13.8)$

$90(56.6)$

$1(0.6)$

$15(9.4)$

7.5
10.1
9.4
3.7

78
49.1
57.9
23.3
25.2

59.7
49.7
17.6
47.8
95
37.6

$1.311^{\#}$

$1.171^{\#}$

$1.215^{\#}$

0.16

62.3

14.5

23.3

108.8

12.3
ProADM group

Total

$\begin{array}{cc}154 & 313 \\ 63.7 & 62.5 \\ 96(62.3) & 190(60.7) \\ 124(80.6) & 250(79.9)\end{array}$

$30(19.4)$

$63(20.1)$

$1.4,1$

$1.3,1$

195

93
37

$24 \quad 48$

2,2

36

73

45

17

59

17

6

87

$2,2^{\#}$

$84^{\#}$

$136^{\#}$

$93^{\#}$

28

121

39

10

161

$\begin{array}{cc}33(21.4) & 64(20.4) \\ 21(13.6) & 43(13.7) \\ 75(48.7) & 165(52.7) \\ 5(3.2) & 6(1.9) \\ 20(13.0) & 35(11.1)\end{array}$

$\begin{array}{ll}1.3 & 4.5\end{array}$

$\begin{array}{ll}7.8 & 8.9\end{array}$

$\begin{array}{ll}7.8 .4 & 9.9 \\ 3.6 & 3.7\end{array}$

$\begin{array}{ll}3.6 & 3.7\end{array}$

$89-83.4$

$55.5-50.4$

$55.5-56.5$

$25.8 \quad 24.6$

$28.4-26.8$

$54.8 \quad 57.2$

$60 \quad 54.6$

$23.9-20.4$

44.5

$94 \quad 95$

$\begin{array}{ll}38 & 37.8\end{array}$

1.599

$1.285-1.241^{\#}$

$1.396 \quad 1.293^{\#}$

$\begin{array}{ll}0.18 & 0.18\end{array}$

$63.6 \quad 62.9$

$\begin{array}{ll}63.6 .3 & 13.4\end{array}$

$24 \quad 23.6$

$107.5-107.2$

$11.5 \quad 11.8$

Data are presented as $n$, unless otherwise stated. ProADM: proadrenomedullin; CURB-65: confusion, urea $>7 \mathrm{mmol} \cdot \mathrm{L}^{-1}$, respiratory rate $\geqslant 30$ breaths $\cdot \mathrm{min}^{-1}$, blood pressure $<90 \mathrm{mmHg}$ (systolic) or $\leqslant 60 \mathrm{mmHg}$ (diastolic), age $\geqslant 65$ years; AECOPD: acute exacerbations of chronic obstructive pulmonary disease; CAP: community-acquired pneumonia. * : values were determined by batch analysis post hoc, and were not known at the time of enrolment and were not available for medical care. 
presentation and $52(32.7 \%)$ after hospital admission; in the proADM group the algorithm was overruled in $42(27.3 \%)$ patients on initial presentation $(\mathrm{p}<0.001)$ and $57(37.0 \%)$ after hospital admission $(\mathrm{p}=0.42)$.

\section{Overruling criteria}

The reasons for overruling on initial presentation were considered medical in 111 (90.2\%) subjects, biopsychosocial and functional in two (1.6\%), organisational in five $(4.1 \%)$ and patient's preference in five $(4.1 \%)$. After reaching medical stability, the triage algorithm was overruled 108 times in the 313 patients $(34.5 \%)$ with consecutively delayed discharge from hospital. The reasons for overruling were considered medical in $27.8 \%$, biopsychosocial and functional in $6.5 \%$, organisational in $52.8 \%$ and patient's preference in $11.1 \%$ (fig. 3). "Waiting for laboratory results, imaging studies or consultant examinations" was the most frequent organisational overruling criterion $(54.4 \%$ of organisational reasons and $28.7 \%$ of all overrulings). The most frequent medical and biopsychosocial/functional overruling criteria stated were "acute other medical problems" and "deficit of mobility or self-care requiring treatment", respectively.

\section{Patients' preferences}

12 patients disliked their initially recommended triage suggestion (five in the control and seven in the proADM group), primarily due to concern about safety at home, and were triaged according to their own preferences. Thus, patient compliance with triage suggestions was $96.2 \%$.

\section{Length of hospital stay}

Overall, mean (95\% confidence interval) LOS was 6.5 days (5.8-7.3 days). 63 (20\%) patients were treated as outpatients, with no difference between the control $(20.7 \%)$ and the proADM group $(19.4 \% ; \mathrm{p}=0.78)$. Mean (95\% CI) LOS in the control group was 6.8 days (5.7-7.9 days) compared with 6.3 days (5.4-7.2 days) in the proADM group. After adjusting for age, sex, LRTI type and CURB-65 score, the proADM group tended to have a shorter LOS (regression coefficient $-0.19,95 \%$ CI $-0.41-0.04 ; \mathrm{p}=0.1$ ). Results were similar when only considering inpatients treated for $\geqslant 1$ day in the hospital $(-0.12,-0.29-0.04$; $p=0.15)$. The results were robust in subgroup analyses without evidence for effect modification ( $\mathrm{p}$ interaction for each subgroup analysis $>0.05$ ) (fig. 4). A similar trend for lower LOS was found when considering the total number of days hospitalised within 90 days after enrolment: 7.9 days (6.7-9.1 days) in the control versus 7.2 days (6.2-8.2 days) in the proADM group (adjusted regression coefficient -0.17 , $-0.40-0.05 ; p=0.13)$. Results were also robust in a per-protocol analysis excluding nonevaluable cases, violators of exclusion criteria and patients with other final diagnoses than LRTI, and when restricted to patients without organisational, biopsychosocial or preference overruling criteria.

The mean time spent in the NLU was 8.1 days (range 1-25 days, median 5.5 days) in patients in the control group $(n=14)$ and 4.5 days (range $2-7$ days, median 4.5 days) in patients in the proADM group $(n=4 ; \mathrm{p}=0.11)$.

\section{Adverse events}

Overall, $21.8 \%$ and $34.5 \%$ of patients experienced an adverse outcome within 30 days and 90 days, respectively. There was no difference between the control and the proADM group for the combined adverse outcome end-point at 30 days (odds ratio $0.81,95 \%$ CI $0.46-1.42 ; \mathrm{p}=0.49$ ) and at 90 days (OR 0.82 , $95 \%$ CI $0.50-1.35 ; \mathrm{p}=0.42$ ). No increased risk was found with regard to mortality, ICU admission and recurrent infection (table 2). Patients' subjective health status on discharge from hospital, measured using the EuroQol-5D visual analogue scale (VAS), was similar in both groups $(61.8 \%$ in control versus $60.3 \%$ in proADM group).

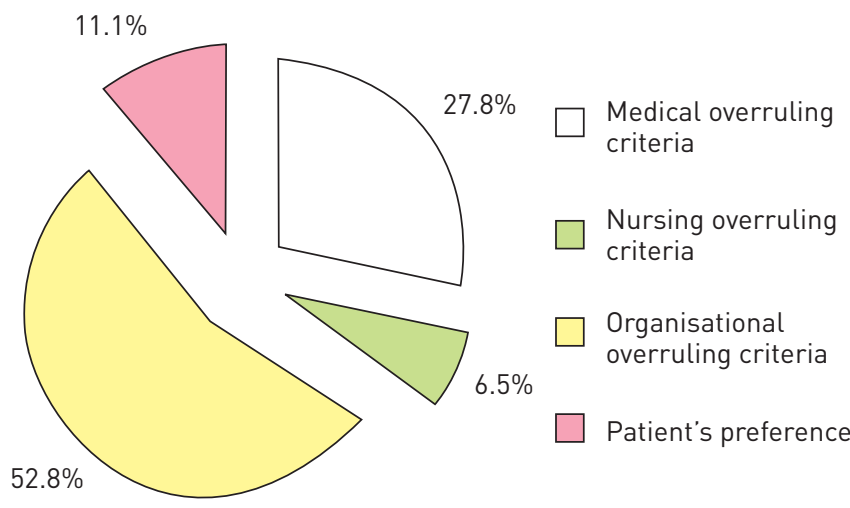

FIGURE 3 Reasons indicated by the treating physician, the nurse in charge or the patient as responsible for overruling of triage after first medical stabilisation. 
FIGURE 4 Subgroups analysis for effect of proadrenomedullin-enhanced triage. Results from the generalised linear model adjusted for age, sex, type of lower respiratory tract infection and severity (according to the confusion, urea $>7 \mathrm{mmol} \cdot \mathrm{L}^{-1}$, respiratory rate $\geqslant 30$ breaths $\cdot \min ^{-1}$, blood pressure $<90 \mathrm{mmHg}$ (systolic) or $\leqslant 60 \mathrm{mmHg}$ (diastolic), age $\geqslant 65$ years (CURB-65) score). CAP: community-acquired pneumonia; LOS: length of stay.

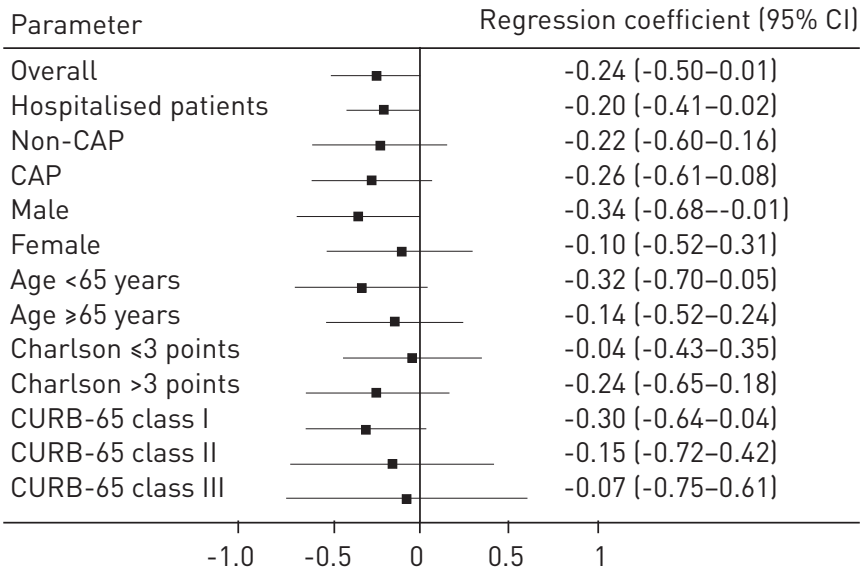

Intervention has shorter LOS Intervention has longer LOS

\section{Readmission rate}

Readmission rates for patients discharged alive within 30 or 90 days were similar in the control group $(8.0 \%$ and $13.3 \%$, respectively) and the proADM group ( $4.9 \%$ and $10.5 \%$, respectively) $(\mathrm{p}=0.29$ and $\mathrm{p}=0.47$ for 30and 90 days, respectively).

\section{Effect of proADM on triage during hospitalisation}

In a large proportion of patients in the proADM group, the proADM values did not change between categories (i.e. values remained $<0.75 \mu \mathrm{g} \cdot \mathrm{L}^{-1}, 0.75-1.5 \mu \mathrm{g} \cdot \mathrm{L}^{-1}$ or $>1.5 \mu \mathrm{g} \cdot \mathrm{L}^{-1}$ ) from admission to day $3(69.9 \%)$ and from day 3 to day $6(72.2 \%$ ) (table 3 ). In $22.1 \%$ and $22.8 \%$, there was a decrease in proADM categories from admission to day 3 and from day 3 to day 6 , respectively, and the proADM category increased in only $8.0 \%$ and $5.1 \%$, respectively.

In the proADM group, 114 and 81 patients were still hospitalised on day 3 and day 6 and, of these, $84.2 \%$ and $79.0 \%$, respectively, were clinically not stable. For patients meeting all stability criteria, a high proADM level indicated that ongoing hospitalisation was necessary in nine (50.0\%) out of 18 on day 3 and in 12 (70.6\%) out of 17 patients on day 6 in the proADM group (table 4).

\section{Historical comparison}

The results of this study were compared with an historical control patient population (OPTIMA I study) [28]. Compared with historical controls, significantly more patients $(20.1 \%$ versus $8.7 \%$; $\mathrm{p}<0.001)$ were treated as outpatients during OPTIMA II. The overall LOS (6.5 days) was 1.9 days (22.6\%) shorter in this current interventional OPTIMA II study compared with an overall LOS of 9.8 days (corresponding to 8.4 days with the same LOS definition of this study) during the OPTIMA I study, i.e. when triage decisions

TABLE 2 Adverse events within 30 and 90 days after enrolment

Adjusted OR $(95 \% \mathrm{Cl})$

p-value
Control group

$35 / 159(22.1)$
$12 / 159(7.6)$
$8 / 159(5.0)$
$5 / 159(3.1)$
$16 / 159(10.1)$

$57 / 159(35.9)$
$14 / 159(8.8)$
$15 / 159(9.4)$
$30 / 159(18.9)$

ProADM group

$31 / 154(20.1)$

$11 / 154(7.1)$

$10 / 154(6.5)$

$5 / 154$ (3.3)

13/154 (8.4)

51/154 (33.1)

$16 / 154$ (10.4)

$13 / 154(8.4)$

27/154 (17.5)

Short-term 30-day outcomes
Any adverse event
Mortality
ICU admission
Recurrent infection
Re-hospitalisation
Long-term $\mathbf{9 0 - d a y ~ o u t c o m e s ~}$
Any adverse event
Mortality
Recurrent infection
Re-hospitalisation

Data are presented as $\mathrm{n} / \mathrm{N}(\%)$, unless otherwise stated. ProADM: proadrenomedullin; ICU: intensive care unit. Patients may experience $>1$ adverse outcome, thus the outcome may total $>100 \%$. 
TABLE 3 Change in proadrenomedullin (proADM) categories over time in the proADM group

Day 1 to day 3

Day 3 to day 6

\begin{tabular}{lcc}
\hline & & \\
No change in proADM category & $79(69.9)$ & $57(72.2)$ \\
ProADM decreasing category & $25(22.1)$ & $18(22.8)$ \\
ProADM increasing category & $9(8.0)$ & $4(5.1)$ \\
\hline
\end{tabular}

Data are presented as $\mathrm{n}(\%)$.

in the same hospital were recommended based on CURB-65 and medical stability criteria but were not reinforced [28]. The effect was even more pronounced in the proADM group (2.1 days, 25.0\%).

\section{Discussion}

While many studies have evaluated the potential of various clinical disease severity scores and biomarkers to improve prognosis in patients with LRTIs, they have all used retrospective or observational designs [33, 34]. However, today's major challenge in view of limited health care resources is to implement these "promising" observational findings into clinical practice. Measuring biomarkers or calculating clinical scores is costly, time-consuming and arguably useless, unless they have a relevant impact on patient care. Based on observational studies, several biomarkers predict adverse outcomes and mortality in patients with LRTIs. These include PCT, which had comparable prognostic accuracy to predict 28-day mortality in CAP as the CURB-65 score [35], and which particularly helps to estimate the risk for treatment failure and mortality if measured serially [36, 37]. However, more investigational biomarkers such as cortisol, proatrial natriuretic peptide, D-dimer, proendothelin-1, copeptin and proADM have shown a higher prognostic accuracy compared with PCT particularly when measured on admission [33, 38]. Even though these biomarkers are rarely compared head-to-head, currently proADM is considered the best single prognostic biomarker $[22,23]$, whereas PCT is currently the best and only systematically evidence-based biomarker to guide antibiotic therapy for patients with LRTI [39].

In this context, we undertook a major effort to assess obstacles to implementing a biomarker-enhanced scoring system into daily practice with an adequate and strong control group in a state-of-the-art randomised controlled trial in order to inform decision makers. Notably, we used a multimodal triage bundle in both groups for individualised interprofessional risk assessment. Our study is unique in being the first randomised controlled proof-of-concept intervention trial to investigate the feasibility of adding the biomarker proADM to established and guideline-recommended clinical criteria for site of care decisions in patients with LRTIs. Although we could not show a significant improvement in main outcome between control and intervention group (evidence level $1 \mathrm{~b}$ ), we found a shorter LOS for patients in this intervention study, regardless of group assignment, and a more pronounced LOS for the proADM group compared with historical controls in the same hospital, which was a pre-defined analysis of evidence level 2b [28].

Indeed, our randomised clinical trial showed a nonsignificant trend for reduction of LOS during the initial encounter (point estimate 0.5 days) and for overall hospitalisations (point estimate 0.7 days) within 90 days in the proADM-enhanced compared with the control group. The reduction of LOS was not achieved through a compensatory increase in days spent in the NLU. In fact, our data do not suggest that the LOS in the NLU was increased using proADM-enhanced triage. The major obstacles were organisational insufficiencies, which are currently typical for many healthcare settings such as the one in Switzerland, e.g. full bed capacity at receiving centres or at night and during weekends, which prevented better adherence to recommended triage pathways in both the proADM and the control group. Patients who had reached

TABLE 4 Influence of high proadrenomedullin (proADM) values on triage decisions in the proADM group

\begin{tabular}{|c|c|c|}
\hline Patients hospitalised & 114 & 81 \\
\hline High proADM indicates nonstability in otherwise medically stable patients & $9 / 18(50.0)$ & $12 / 17(70.6)$ \\
\hline
\end{tabular}

Data are presented as $\mathrm{n}$ or $\mathrm{n} / \mathrm{N}(\%)$. 
medical stability but required additional nursing care or rehabilitation frequently remained hospitalised due to limited capacities at receiving rehabilitative institutions. Furthermore, many patients required hospitalisation for medical problems unrelated to the LRTI. On admission, when $90 \%$ of overrulings were for medical reasons, overruling was significantly more frequent in the control than the proADM group.

While our study apparently was underpowered and the significance level of proADM-enhanced triage was formally missed, the trend of our findings was robust and unequivocal in all examined subgroups. A reduction of 0.5 days (within 30 days of follow-up; 7.3\%) and 0.7 days (within 90 days of follow-up; $8.9 \%$ ) for the proADM groups is clinically remarkable as it was embedded in an interdisciplinary risk assessment bundle and compared with a highly competitive, guideline-conforming and strictly reinforced control group.

Thus, our results must be interpreted in light of organisational challenges. In such a setting with a strictly reinforced control group and major logistical hurdles, we consider the results of this study clinically relevant. The impact of the proADM-enhanced algorithm is therefore expected in settings with facilitated transition mechanisms to nonacute medical care.

Our interdisciplinary, multimodal approach led to outpatient treatment in $\sim 20 \%$ of patients, which is more than double that of our historical control (9\%) [28]. This achievement alone confers the advantages of reduction of costs [8] and of nosocomial infections [40]. Patients treated at home resume normal activities sooner and experience fewer thromboembolic events [40]. Risk of worsening of pre-existing frailty or delirium is lower in outpatients. One-third of elderly patients develop disabilities after hospitalisations for medical reasons such as CAP [41], as 50\% of disabilities in elderly persons occur in the setting of hospitalisation [42]. If given the choice, most low-risk patients with CAP prefer outpatient care [43], which is associated with similar outcomes but higher patient satisfaction and lower costs [44].

A 0.5-day shorter LOS was estimated to correspond to $\$ 1.37$ billion annual savings for CAP patients in the US [45]. Interestingly, this equals our point estimate of shortening of LOS, even though it did not reach statistical significance.

Clinical pathway bundles in the management of pneumonia reduced hospitalisation rate and LOS particularly in low-risk patients, and decreased overall costs while achieving similar quality of life and patient outcomes [46-48]. These interventions were complex and resource-intensive. Recently, a three-step critical pathway (early mobilisation, criteria for switching to oral antibiotics and objective criteria regarding the need for hospital care or discharge, i.e. the core of our algorithm) was found effective and safe to reduce both length of antibiotic treatment and LOS [44]. Our triage algorithm attempts to be both comprehensive and feasible for real-life. It takes medical, functional or social aspects into account and therefore consists of several different risk assessments and predefined overruling criteria. Despite its complexity, we have been able to implement it in our hospital into clinical routine since the end of this study with the help and dedication of the medical and nursing departments and our social workers.

The CURB-65 score has also previously been shown to be applicable to patients with non-CAP LRTI [16-18]. While the medical stability criteria [30], which were used in this trial, were not formally extended to patients with non-CAP LRTI, there is little reason to limit their use to CAP considering the fact that many patients are, allegedly unnecessarily, hospitalised without formal infiltrate on chest radiograph but with symptoms of LRTI, i.e. acute bronchitis and AECOPD.

Importantly, there was no increased risk for readmissions or serious adverse events and patients reported similar health status on hospital discharge between the two groups. Fear of medical complications has previously been shown to be the most important cause for hospitalisation [6]. This was independent of disease severity, as indicated by clinical risk scores, and it did not correlate with successful PCT-guided antibiotic stewardship [49]. A 96.2\% patients' agreement with the triage algorithm also confirms that patients feel safe and comfortable with triage decisions. Our proADM algorithm was not designed to result in earlier discharges than would be theoretically possible with CURB-65 and medical stability criteria alone. Instead, proADM provided an additional safety tool to increase confidence in readiness for discharge. In fact, high proADM values on day 3 and day 6 led to ongoing hospitalisations in $50 \%$ and $70 \%$ of hospitalised patients, respectively, who otherwise were medically stable and ready for discharge according to clinical criteria alone.

One of the strengths of this study was its innovative design. The functional assessment was evidence based [12], the biomarker-enhanced risk score was derived and validated based on a large multicentre randomised controlled clinical trial (ProHOSP) [16] and an additional observational study (OPTIMA I) [28]. Furthermore, this study is timely and relevant in view of the increasingly wide-spread implementation of diagnosis-related groups (DRGs) where an effective triage and a timely discharge will be of great importance. It is important to apply evidence-based triage algorithms to prevent unwanted complications. 
Although the effect of proADM guidance missed statistical significance, it is noteworthy that the control group had a short LOS due to very efficient triage based on strongly reinforced guideline recommendations, thus correcting for a Hawthorne limitation, a known limitation of previous projects in biomarker research. Other contributing factors were organisational overrulings, as the Swiss healthcare system is not yet that well prepared for early discharges. Additional strengths were the high recruitment rate and low loss to follow-up. Finally, due to the objective triage criteria used, our triage algorithm might be applied to other healthcare systems. However, its feasibility and utility need to be confirmed in other optimised settings and larger patient populations.

In conclusion, in this proof-of-concept trial, we show the feasibility and challenges of an interdisciplinary and biomarker-enhanced triage algorithm to shorten LOS in patients with LRTIs and thereby avoid medically unnecessary days in the hospital. In settings with broader opportunities for outpatient and nonacute hospital care, the benefit of a structured risk assessment might be even larger.

\section{Acknowledgements}

We are indebted to Werner Zimmerli (Medical University Department, Kantonsspital Liestal, Switzerland), Andre Perruchoud (Riehen, Switzerland) and Nicolas Garin (University Hospital Geneva, Switzerland) as the members of the Data Safety and Monitoring Board (Kantonsspital Aarau, Aarau, Switzerland).

We are grateful to Renate Hunziker and the staff of the central medical laboratory of the Kantonsspital Aarau for their expert technical and logistic support; to Petra Tobias and Susanne Schirlo and the medical and nursing staff of the Emergency Dept and the medical wards of the Kantonsspital Aarau for their support and participation; to Ruth Schweingruber and Anja Keller for logistic support; to Martin Frey, Lukas Schlatter, Rene Fiechter, Beat Stierlin and the medical and nursing staff of the Klinik Barmelweid (Barmelweid, Switzerland) for their support; to Stefan Mariacher, Eveline Wiederkehr and the medical and nursing staff of the AarReha Schinznach (Schinznach-Bad, Switzerland) for their support; and to Jörg Fröhlich, Renata Kleeb and Martin Flückiger (all Kantonsspital Aarau) for expert financial advice. The authors are grateful to Martin Roth, Theres Matter and Susanne Hochuli of the Dept for Gesundheit und Soziales, Kanton Aargau, for financial support.

\section{References}

Grijalva CG, Nuorti JP, Arbogast PG, et al. Decline in pneumonia admissions after routine childhood immunisation with pneumococcal conjugate vaccine in the USA: a time-series analysis. Lancet 2007; 369: 1179-1186.

2 Aliyu ZY, Aliyu MH, McCormick K. Determinants for hospitalization in " low-risk" community acquired pneumonia. BMC Infect Dis 2003; 3: 11.

3 Griffiths PD, Edwards MH, Forbes A, et al. Effectiveness of intermediate care in nursing-led in-patient units. Cochrane Database Syst Rev, 2007: CD002214.

4 Chalmers JD, Al-Khairalla M, Short PM, et al. Proposed changes to management of lower respiratory tract infections in response to the Clostridium difficile epidemic. J Antimicrob Chemother 2010; 65: 608-618.

5 Aujesky D, McCausland JB, Whittle J, et al. Reasons why emergency department providers do not rely on the pneumonia severity index to determine the initial site of treatment for patients with pneumonia. Clin Infect Dis 2009; 49: e100-e108.

6 Baehni C, Meier S, Spreiter P, et al. Which patients with lower respiratory tract infections need inpatient treatment? Perceptions of physicians, nurses, patients and relatives. BMC Pulm Med 2010; 10: 12.

7 Choudhury G, Chalmers JD, Mandal P, et al. Physician judgement is a crucial adjunct to pneumonia severity scores in low-risk patients. Eur Respir J 2011; 38: 643-648.

8 Labarere J, Stone RA, Scott Obrosky D, et al. Factors associated with the hospitalization of low-risk patients with community-acquired pneumonia in a cluster-randomized trial. J Gen Intern Med 2006; 21: 745-752.

9 McGregor MJ, Fitzgerald JM, Reid RJ, et al. Determinants of hospital length of stay among patients with pneumonia admitted to a large Canadian hospital from 1991 to 2001. Can Respir J 2005; 12: 365-370.

10 Menéndez R, Cremades MJ, Martínez-Moragón E, et al. Duration of length of stay in pneumonia: influence of clinical factors and hospital type. Eur Respir J 2003; 22: 643-648.

11 Schlarmann J. Der CMS im ePA. Verschiedene Qualitätsdimensionen eines Instruments. Eine empirische Analyse. [The CMS in ePA. Diverse Quality Dimensions of an Instrument. An Empirical Analysis.] PhD Thesis. Universität Witten/Herdecke, Gelsenkirchen, Germany, 2007.

12 Louis Simonet M, Kossovsky MP, Chopard P, et al. A predictive score to identify hospitalized patients' risk of discharge to a post-acute care facility. BMC Health Serv Res 2008; 8: 154.

13 Griffiths P, Edwards M, Forbes A, et al. Post-acute intermediate care in nursing-led units: a systematic review of effectiveness. Int J Nurs Stud 2005; 42: 107-116.

14 Griffiths P, Wilson-Barnett J. The effectiveness of "nursing beds": a review of the literature. J Adv Nurs 1998; 27: $1184-1192$.

15 Woodhead M, Blasi F, Ewig S, et al. Guidelines for the management of adult lower respiratory tract infections-full version. Clin Microbiol Infect 2011; 17: Suppl. 6, E1-E59.

16 Albrich WC, Dusemund F, Ruegger K, et al. Enhancement of CURB65 score with proadrenomedullin (CURB65-A) for outcome prediction in lower respiratory tract infections: derivation of a clinical algorithm. BMC Infect Dis 2011; 11: 112 .

17 Chang CL, Sullivan GD, Karalus NC, et al. Predicting early mortality in acute exacerbation of chronic obstructive pulmonary disease using the CURB65 score. Respirology 2011; 16: 146-151.

18 Schuetz P, Koller M, Christ-Crain M, et al. Predicting mortality with pneumonia severity scores: importance of model recalibration to local settings. Epidemiol Infect 2008; 136: 1628-1637. 
19 Huang DT, Weissfeld LA, Kellum JA, et al. Risk prediction with procalcitonin and clinical rules in communityacquired pneumonia. Ann Emerg Med 2008; 52: 48-58.

20 Christ-Crain M, Morgenthaler NG, Stolz D, et al. Pro-adrenomedullin to predict severity and outcome in community-acquired pneumonia [ISRCTN04176397]. Crit Care 2006; 10: R96.

21 Huang DT, Angus DC, Kellum JA, et al. Midregional proadrenomedullin as a prognostic tool in communityacquired pneumonia. Chest 2009; 136: 823-831.

22 Krüger S, Ewig S, Giersdorf S, et al. Cardiovascular and inflammatory biomarkers to predict short- and long-term survival in community-acquired pneumonia: results from the German Competence Network, CAPNETZ. Am J Respir Crit Care Med 2010; 182: 1426-1434.

23 Schuetz $\mathrm{P}$, Wolbers M, Christ-Crain M, et al. Prohormones for prediction of adverse medical outcome in community-acquired pneumonia and lower respiratory tract infections. Crit Care 2010; 14: R106.

24 Ronan D, Nathwani D, Davey P, et al. Predicting mortality in patients with community-acquired pneumonia and low CURB-65 scores. Eur J Clin Microbiol Infect Dis 2010; 29: 1117-1124.

25 Müller B, Morgenthaler N, Stolz D, et al. Circulating levels of copeptin, a novel biomarker, in lower respiratory tract infections. Eur J Clin Investig 2007; 37: 145-152.

26 Müller B, Süess E, Schuetz P, et al. Circulating levels of pro-atrial natriuretic peptide in lower respiratory tract infections. J Intern Med 2006; 260: 568-576.

27 Schuetz P, Stolz D, Mueller B, et al. Endothelin-1 precursor peptides correlate with severity of disease and outcome in patients with community acquired pneumonia. BMC Infect Dis 2008; 8: 22.

28 Albrich WC, Rüegger K, Dusemund F, et al. Optimised Patient Transfer using an Innovative Multidisciplinary Assessment in Kanton Aargau (OPTIMA I): an observational survey in lower respiratory tract infections. Swiss Med Wkly 2011; 141: w13237.

29 Chalmers JD, Singanayagam A, Akram AR, et al. Severity assessment tools for predicting mortality in hospitalised patients with community-acquired pneumonia. Systematic review and meta-analysis. Thorax 2010; 65: 878-883.

30 Mandell LA. Severe community-acquired pneumonia (CAP) and the Infectious Diseases Society of America/ American Thoracic Society CAP guidelines prediction rule: validated or not. Clin Infect Dis 2009; 48: 386-388.

31 Mandell LA, Wunderink RG, Anzueto A, et al. Infectious Diseases Society of America/American Thoracic Society consensus guidelines on the management of community-acquired pneumonia in adults. Clin Infect Dis 2007; 44: Suppl. 2, S27-S72.

32 Dodd S, Bassi A, Bodger K, et al. A comparison of multivariable regression models to analyse cost data. J Eval Clin Pract 2006; 12: 76-86.

33 Christ-Crain M, Opal SM. Clinical review: the role of biomarkers in the diagnosis and management of communityacquired pneumonia. Crit Care 2010; 14: 203.

34 Krüger S, Frechen D. Another brick in the wall: adrenomedullin and prognosis in community-acquired pneumonia. Eur Respir J 2012; 39: 1060-1061.

35 Krüger S, Ewig S, Marre R, et al. Procalcitonin predicts patients at low risk of death from community-acquired pneumonia across all CRB-65 classes. Eur Respir J 2008; 31: 349-355.

36 Harbarth S, Holeckova K, Froidevaux C, et al. Diagnostic value of procalcitonin, interleukin-6, and interleukin-8 in critically ill patients admitted with suspected sepsis. Am J Respir Crit Care Med 2001; 164: 396-402.

37 Jensen JU, Heslet L, Jensen TH, et al. Procalcitonin increase in early identification of critically ill patients at high risk of mortality. Crit Care Med 2006; 34: 2596-2602.

38 Krüger S, Welte T. Biomarkers in community-acquired pneumonia. Expert Rev Respir Med 2012; 6: 203-214.

39 Schuetz P, Müller B, Christ-Crain M, et al. Procalcitonin to initiate or discontinue antibiotics in acute respiratory tract infections. Cochrane Database Syst Rev 2012; 9: CD007498.

40 Mandell L. Decisions about treating community-acquired pneumonia. Ann Intern Med 2005; 142: 215-216.

41 Covinsky KE, Pierluissi E, Johnston CB. Hospitalization-associated disability: "she was probably able to ambulate, but I'm not sure". JAMA 2011; 306: 1782-1793.

42 Gill TM, Allore HG, Holford TR, et al. Hospitalization, restricted activity, and the development of disability among older persons. JAMA 2004; 292: 2115-2124.

43 Coley CM, Li YH, Medsger AR, et al. Preferences for home vs hospital care among low-risk patients with community-acquired pneumonia. Arch Intern Med 1996; 156: 1565-1571.

44 Carratalà J, Garcia-Vidal C, Ortega L, et al. Effect of a 3-step critical pathway to reduce duration of intravenous antibiotic therapy and length of stay in community-acquired pneumonia: a randomized controlled trial. Arch Intern Med 2012; 172: 922-928.

45 Raut M, Schein J, Mody S, et al. Estimating the economic impact of a half-day reduction in length of hospital stay among patients with community-acquired pneumonia in the US. Curr Med Res Opin 2009; 25: 2151-2157.

46 Chalmers JD, Akram AR, Hill AT. Increasing outpatient treatment of mild community-acquired pneumonia: systematic review and meta-analysis. Eur Respir J 2010; 37: 858-864.

47 Loeb M, Carusone SC, Goeree R, et al. Effect of a clinical pathway to reduce hospitalizations in nursing home residents with pneumonia: a randomized controlled trial. JAMA 2006; 295: 2503-2510.

48 Marrie TJ, Lau CY, Wheeler SL, et al. A controlled trial of a critical pathway for treatment of community-acquired pneumonia. CAPITAL study investigators. Community-acquired pneumonia intervention trial assessing levofloxacin. JAMA 2000; 283: 749-755.

49 Schuetz P, Christ-Crain M, Thomann R, et al. Effect of procalcitonin-based guidelines vs standard guidelines on antibiotic use in lower respiratory tract infections: the ProHOSP randomized controlled trial. JAMA 2009; 302: 1059-1066. 